\title{
Beating in the RHEED Intensity Oscillations during Surfactant Mediated GaAs Molecular Beam Epitaxy: Process Physics and Modeling
}

\begin{abstract}
VAMSEE K. PAMULA and R. VENKAT
Department of Electrical and Computer Engineering, University of Nevada, Las Vegas, Las Vegas, NV 89154-4026

In a recent work, beating in the reflection high energy electron diffraction (RHEED) intensity oscillations were observed during molecular beam epitaxial (MBE) growth of $G a A s$ with $S n$ as a surfactant. The strength of beating is found to be dependent on the $S n$ submonolayer coverage with strong beating observed for 0.4 monolayer coverage. For a fixed temperature and flux ratio ( $G a$ to $A s)$, the period of oscillation decreases with increasing $S n$ coverage. In this work, we have developed a rate equation model of growth to investigate this phenomenon. In our model, the $G a A s$ covered by the $S n$ is assumed to grow at a faster rate compared to the $\mathrm{GaAs}$ not covered by $\mathrm{Sn}$. Assuming that the electron beams reflected from the $\mathrm{Sn}$ covered surface and the rest of the surface are incoherent, the results of the dependence of the RHEED oscillations on $S n$ submonolayer coverages for various $S n$ coverages were obtained and compared with experimental data and the agreement is good.
\end{abstract}

Keywords: Surfactant, GaAs, MBE, RHEED, Modeling, Rate Equation.

\section{INTRODUCTION}

The presence of impurities on the surface is known to influence the flow of steps during the growth and modify the surface roughness, the composition of the epilayer and the degree of ordering [1-3]. In the growth of $G a A s, S n$, which is an amphoteric dopant is known to ride the surface due to much larger atomic radius of $S n\left(1.42 A^{\circ}\right)$ compared to that of $G a\left(1.22 A^{\circ}\right)$ and $A s\left(1.18 A^{\circ}\right)$. Petrich et al [4] have experimentally studied the effect of submonolayer concentrations of $S n$ on the surface and observed the occurrence of beating in the RHEED intensity oscillations of the specular spot. They observed that the $S n$ submonolayer coverage required for strong beating decreases with temperature. They also observed that at $600^{\circ} \mathrm{C}$, the period of oscillation decreases with $S n$ coverage.

In this article, we propose a kinetic rate equation model to explain the observed beating in the RHEED intensity oscillations of Ref.[4]. The details of the model are presented in in Section 2. Our results are compared with that of experiments of Ref.[4] in Section 3. Conclusions are presented in Section 4. 


\section{THE PROPOSED MODEL}

\subsection{Physics Of The Model}

The presence of beats in the RHEED intensity oscillations (RO's) is assumed to be a result of two different intensity oscillations of slightly different frequencies adding up incoherently. A possible physical growth model which will yield two RO's of different frequencies is as follows. In a surfactant mediated growth with submonolayer coverage of the surfactant there are two distinct surfaces, one covered with the surfactant and the other not so. If the island size of the surfactant covered area is much larger than the coherent length of the incident electron beam, the resultant reflected intensity will be a sum of three components, intensities from surfactant covered surface, interface and surface not covered by the surfactant. The intensity components from the two surfaces will be incoherent if the $S n$ island size is larger than the coherent length of electron beam (about $100 \AA$ for a typical $10 \mathrm{kV}$ RHEED system). If the growth rates for the $S n$ covered and non - $S n$-covered surfaces are different, then the RHEED intensities from these two areas will oscillate at different frequencies with respect to time. If these intensities interfere incoherently, beats will be observed in the resultant intensity. The interfacial component of intensity will be the coherent component which will contribute insignificantly to the total intensity for large island sizes because as the island size increases, the interfacial area (coherent component) to bulk area (incoherent component) ratio decreases.

\subsection{The Rate Equation Model}

The only elementary surface kinetic processes included in our model are: adsorption and migration. The time rates of change of concentration of $G a$ and As under the $S n$-covered and non - Sn-covered surfaces can be described in terms of the rates of individual kinetic processes. Thus, the time rate of change of
$S n$-covered $G a$ concentration in the $2 n^{\text {th }}$ layer, $C_{G a}^{S n}(2 n)$, can be written as:

$$
\begin{gathered}
\frac{d C_{G a}^{S n}(2 n)}{d t}=S_{G a} J_{G a}\left[C_{A s}^{S n}(2 n-1)-C_{G a}^{S n}(2 n)\right] \\
+\left[C_{A s}^{S n}(2 n-1)-C_{G a}^{S n}(2 n)\right] \\
\times\left(R_{o, G a} e^{\frac{-E_{G a}^{S n}(2 n+2)}{k T}}\left[C_{G a}^{S n}(2 n+2)-C_{A s}^{S n}(2 n+3)\right]\right. \\
\left.+R_{o, G a} e^{-\frac{E_{G a}^{S n}(2 n-2)}{k T}}\left[C_{G a}^{S n}(2 n-2)-C_{A s}^{S n}(2 n-1)\right]\right) \\
-R_{o, G a} e^{\frac{E_{G a}^{S n}(2 n)}{k T}}\left[C_{G a}^{S n}(2 n)-C_{A s}^{S n}(2 n+1)\right] \\
\times\left(\left[C_{A s}^{S n}(2 n-3)-C_{G a}^{S n}(2 n-2)\right]+\right. \\
\left.\quad\left[C_{A s}^{S n}(2 n+1)-C_{G a}^{S n}(2 n+2)\right]\right)
\end{gathered}
$$

The term $A l$ represents the adsorption of $G a$ atoms on the $S n$-covered surface. The flux and sticking probability of $G a$ are denoted as $J_{G a}$ and $S_{G a}$, respectively. The term $A 2$ describes the rate of addition of $G a$ from adjacent $G a$ layers by migration through Arrhenius type rate equation with activation energy, $E_{G a}^{S n}$, and frequency factor, $R_{o, G a}$. The activation energy for migration of $G a$ atoms from the $(2 n+2)^{t h}$ is given by:

$E_{G a}^{S n}(2 n+2)=E_{i s o, G a}^{S n}(2 n+2)+z E_{G a G a}^{S n} C_{G a}^{S n}(2 n+2)$

and a similar equation is assumed for the $(2 n-2)^{t h}$ layer. In Equation 2, the $E_{i s o, G a}^{S n}$ and $E_{G a, G a}^{S n}$ define the activation energy of migration of an isolated $G a$ on the surface and interaction energy for second nearest neighbor $G a-G a$ pairs, respectively. $z$ is the inplane coordination number for the (100) plane which is 4 . The term A3 in the equation represents the rate of deletion of atoms from $2 n^{\text {th }}$ layer due to migration of $G a$ atoms to adjacent layers. Similar time evolution equations can be written for $G a$ and $A s$ on non-Sn-covered and $A s$ on $S n$-covered surface. Additionally, the total concentration of $G a$ in the $2 n^{t h}$ layer satisfies the following condition :

$$
C_{G a}^{S n}(2 n)+C_{G a}^{n o n-S n}(2 n) \leq 1.0
$$

where $C_{G a}^{n o n-S n}(2 n)$ is the concentration of $G a$ in on the non-Sn-covered area of the $2 n^{\text {th }}$ layer.

The frequency factor, $R_{o}$, for $G a$ and $A s$ were assumed to be $10^{13} / \mathrm{sec}$. $E_{i s o}$ for $G a$ and $A s$ for all sur- 
faces were assumed to be $1.3 \mathrm{eV} . E_{G a G a}$ and $E_{A s A s}$ for both the surfaces is assumed equal to $0.17 \mathrm{eV}$. The sticking probabilities of $G a$ for the two surfaces were assumed to be different and used as fitting parameters of the model.

\section{RESULTS AND DISCUSSION}

The $2 n$ coupled first order non-linear differential equations for $2 n$ layers were solved numerically with a time step of $10^{-6}$ seconds. Instantaneous RHEED intensities of specular spot were computed using the kinematical theory of electron diffraction with a $10 \mathrm{kV}$ electron beam at $1.5^{\circ}$ grazing incidence. The fitting parameters (sticking probability of $\mathrm{Ga}$ on $\mathrm{Sn}$ covered and non-Sn-covered surfaces) were obtained by matching the experimental and theoretical RHEED intensity oscillations (RO's).

Simulations were performed for various submonolayer coverages of $S n$ for a temperature of $600^{\circ} \mathrm{C}$ at a flux rate of 1 monolayer/sec. The RHEED intensity versus time plot is shown in Figure 1. The qualitative agreement between the RO's reported in Ref.[4] with Figure 1 is fairly good. The RO's are dependent on the $S n$ coverage with low and high coverages yielding typical RO's observed in MBE growths and medium coverages resulting in beating behavior as shown in

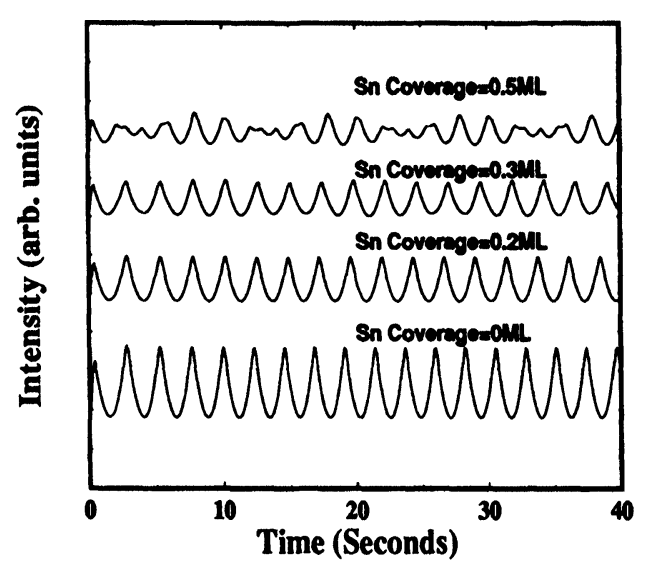

FIGURE 1 RHEED intensity versus time for various submonolayer coverages of $S n$ at $600^{\circ} \mathrm{C}$
Figure 1. The beating behavior is a result of RHEED intensities from the $S n$-covered and non - Sn-covered surfaces adding up incoherently. For low and high $S n$ coverages, the RHEED intensity in the specular beam contains predominantly a single frequency component from either the non - Sn-covered or the $S n$-covered GaAs with both coherent and incoherent components. Since, the frequencies of the coherent and incoherent components are the same, there is no beating observed. However, for medium coverage of $S n$, the RHEED intensity contains three components: intensities from within $S n$-covered and non - $S n$-covered surfaces respectively and electron beam reflected from the interface between these surfaces which is coherent. The reflected electron beams from the $S n$ covered surfaces and from the non-Sn-covered GaAs will not be coherent with each other if the island sizes of $S n$ are larger than the coherent length of the electron beam (which is typically of the order of $100 \AA$ ). The third coherent component arising from the interface will be insignificant for large island sizes as the interfacial area decreases in comparison to the $S n$-covered or non-Sn-covered areas. Thus, for medium $S n$ coverages, the addition of intensities from incoherent electron beams from the $S n$-covered and $n o n-S n$-covered surfaces results. Since these two areas grow at different growth rates, the frequencies of oscillation of these two components will be slightly different which will yield beats in RO's. Additionally, the amplitude of RO's decreased slightly with $S n$ submonolayer coverage as shown in Figure 2 unlike experiments in which the amplitude increased slightly which may be a result of assumptions in the RHEED computation such as exclusion of multiple reflections, atomic scattering factor differences and effect of surface reconstruction on the RHEED intensity.

A plot of time period of the RO's versus $S n$ submonolayer coverages at $600^{\circ} \mathrm{C}$ is shown in Figure 2 . As the $S n$ submonolayer coverage increases, the period of RO decreases which is in good agreement with the experimental data of Ref.[4]. Noting that the period of RO's is inversely related to the growth rate and that the $S n$-covered are a grows at a faster rate compared to non - Sn-covered area, it is expected that for large $S n$ coverages, the average growth rate will be 


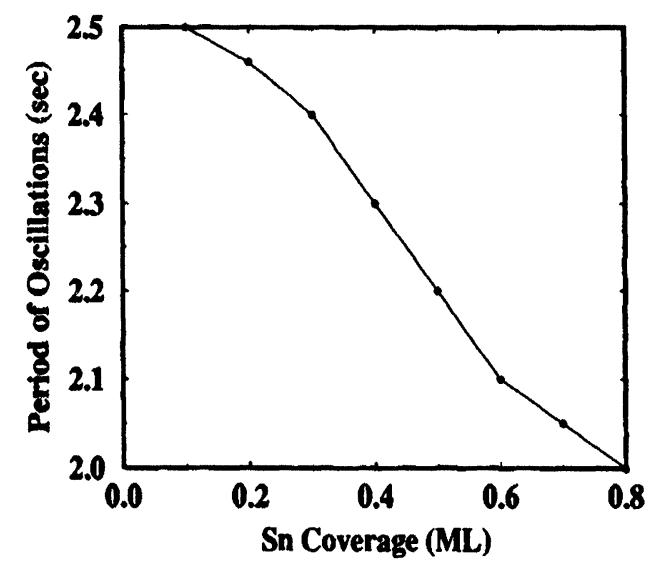

FIGURE 2 Period of RO's for various submonolayer coverages of $S n$ at $600^{\circ} \mathrm{C}$

larger and hence a smaller period of RO's and vice versa.

\section{CONCLUSION}

In this work, a rate equation model is proposed and utilized to investigate surfactant mediated MBE growth of GaAs. The model reproduces semi-quantitatively most of the experimental observations of RHEED oscillations and their dependence on the surface conditions such as the $S n$ coverage. The key assumption of the model is that the growth rates of $G a A s$ on $S n$-covered and non - Sn-covered surfaces can be different and that electron beam intensities reflected from these two surfaces, if separated by more than the coherent length of the electron beam, will interfere incoherently. It was found that the GaAs on $S n$-covered surface grows at faster rate compared to GaAs on non - Sn-covered GaAs.

\section{References}

[1] A.A. Chernov, Solid State Sci. 36, 159 (1984).

[2] M. Copel, M.C. Reuter, M. Horn Von Hoegen, and R.M.Tromp, "Influence of surfactants in Ge and Si epitaxy on Si(001)", Phys. Rev. B. 42, 11682 (1990).

[3] H. Saito, and T. Fukui, "Impurity-induced disordering in fractional-layer growth on a (001) vicinal surface by metalorganic chemical vapor deposition", Appl. Phys. Lett. 56, 87 (1990).

[4] G.S. Petrich, A.M. Dabiran, J.E. Macdonald, and P.I. Cohen, "The effect of submonolayer Sn $\delta$-doping layers on the growth of InGaAs and GaAs", J. Vac. Sci. Technol, B 9, 2150 (1991).

\section{Biographies}

Vamsee $\mathbf{K}$ Pamula is a graduate student in the Department of Electrical Engineering at Duke University. His research interests include semiconductor devices and materials.

R.Venkatasubramanian is an Associate Professor in the Department of Electrical Engineering at University of Nevada, Las Vegas. His research interests include process and device physics and modeling. 

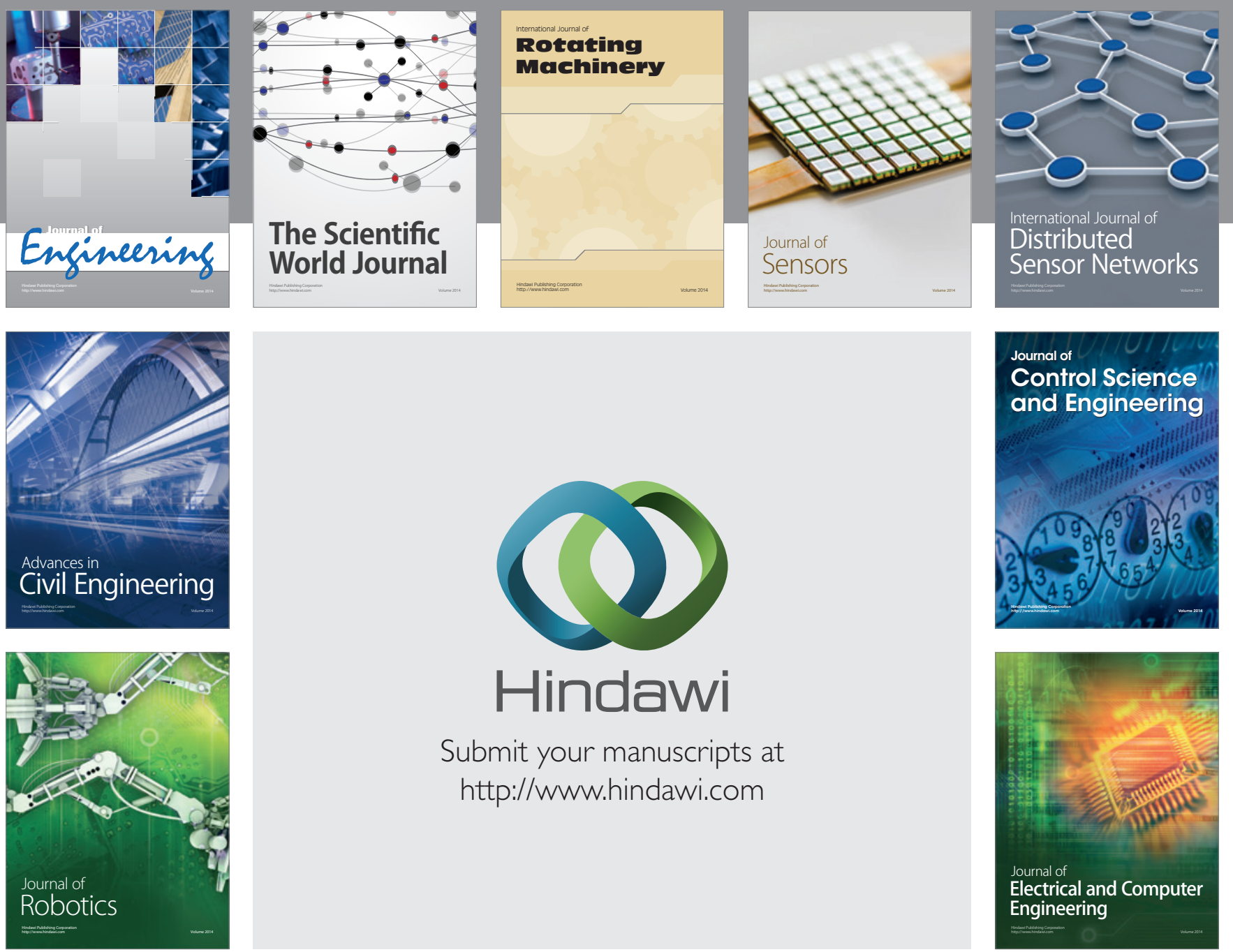

Submit your manuscripts at

http://www.hindawi.com
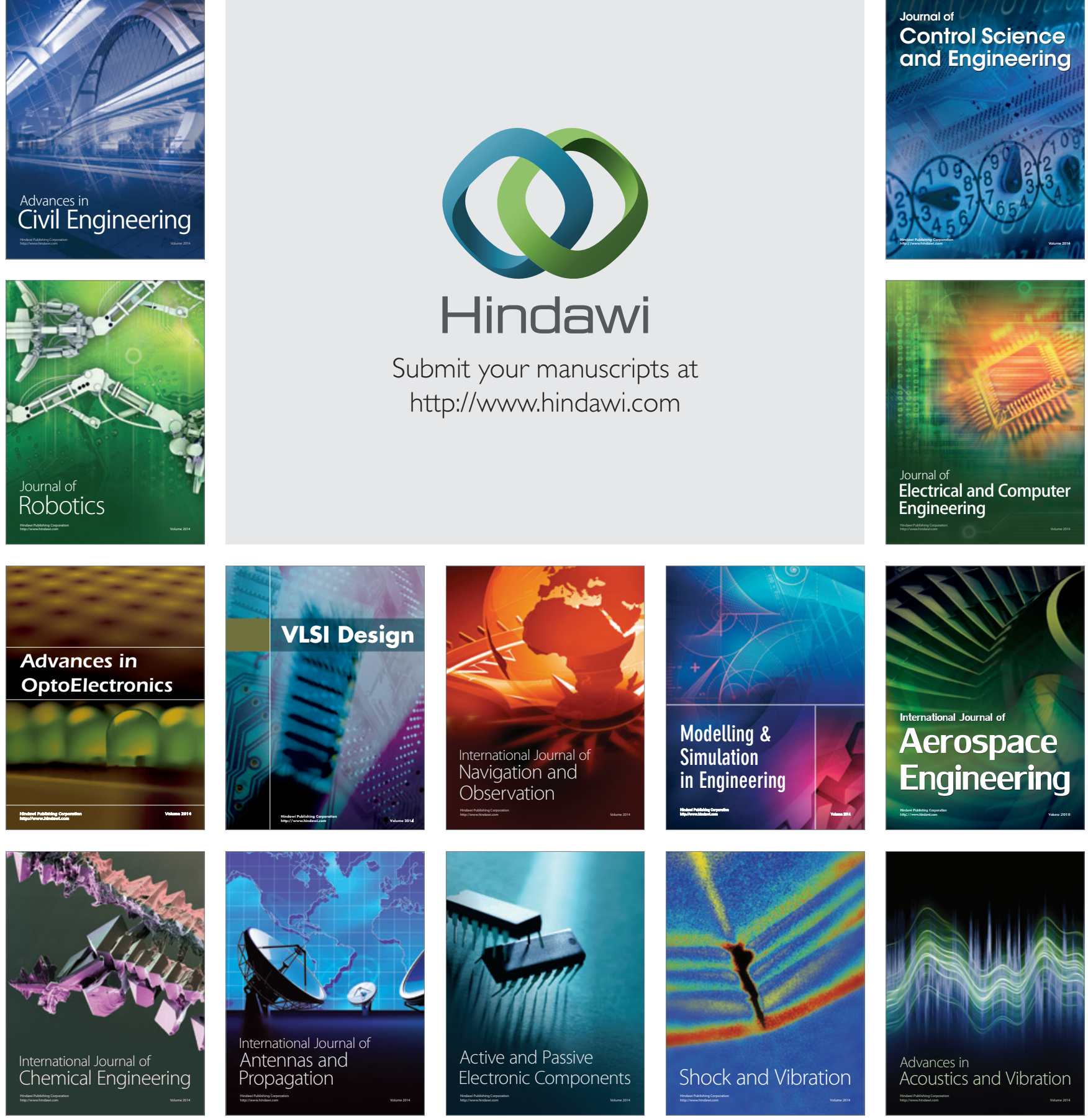